\title{
EDITORIAL
}

\section{Looking inwards}

\author{
The publication of a new article type prompts us to have a look back at the evolution and \\ development of the journal over the past decade.
}

2013 marks Nature Reviews Microbiology's tenth anniversary. As we are fond of noting, over the past decade there have been some amazing developments in microbiology, and doubtless we will wax lyrical about what we regard as the most important in our tenth-anniversary issue in October 2013. As a review journal, our raison dêtre, of course, is to try and reflect the current themes and trends in microbiology research, and keeping up with what's hot and what's not is one of the most important aspects of an editor's job. In this anniversary year, however, it is also instructive for us to take some time to look inwards and reflect on how and why the journal has changed since our first issue.

Members of the editorial team who are of a certain vintage have been around long enough to remember the first issue vividly. The core content of that issue comprised two broad article types: first, high-quality Reviews on hot topics in microbiology, which were commissioned from leaders in the field and peer reviewed, and featured clear and informative figures to illustrate key concepts; and second, a mixture of interesting Perspectives, also commissioned by the editorial team and peer reviewed, and taking the same approach to figure presentation as the Reviews. Since 2003, the scientific publishing landscape has changed dramatically, particularly in the past 5 years, but a quick inspection of the journal over that time shows that our fundamental content is essentially unchanged. We are in the midst of one of the most turbulent periods in scientific publishing since the development of online content, and in time, this turbulence will inevitably begin to buffet review journals. In the meantime, however, most of the informal feedback that the editorial team has gathered at conferences, as well as the more formal feedback that we have obtained through email questionnaires and author surveys, indicates that there is no great desire for a massive shake-up in the content we feature or, for now, in the way in which it is presented.

That's not to say that there haven't been a few important changes along the way, in terms of both the specific article types that we publish, and journal design and functionality. Introducing new article types can sometimes be a question of semantics; after all, few readers (or authors) really care if a Perspective is labelled as an Essay or a Science \& Society article, for example. However, in response to specific requests from the community, two new article types were introduced back in 2007. Analysis articles are Review-type articles that allow authors to use an existing data set, such as metagenomic data, to produce a novel hypothesis or conclusion. Progress articles follow more of a mini-review format, being shorter in length and generally narrower in scope than full Reviews, and thus can be published quickly, allowing us to keep up with the pace of advancement in fast-moving areas of microbiology research.

In addition, a flick through the first issue involves a journey back in typographical time, and there has been one major print and online redesign, way back in 2006. Seven years is a long time to maintain the same template online, and we are delighted that a long-awaited rejig in the online appearance of our articles is due to be rolled out on all of the Nature Reviews journals in the next few months, bringing us in line with Nature and the Nature-branded research journals. The new template brings greater flexibility and will hopefully enable us to begin to make full use of different multimedia elements. In what we hope will be a welcome development, the new design also includes article metrics, so authors and readers will be able to gauge how well an article has 'performed' at a glance. Moreover, along with eight other Nature Publishing Group titles, including Nature, Nature Reviews Microbiology is available within the relaunched Nature Journals iPad app, which is designed to combine the clarity of print with the enhanced functionality you would expect with an app.

Finally, as we reach the advanced age of 10 years old, we have decided to introduce another new article type, the Viewpoint, featuring responses from a panel of experts in a fast-moving or controversial area to a series of questions devised by the editorial team. Rapid change in a research field often goes hand in hand with disagreement and debate on the best way forward, but researchers who hold differing views on a particular topic don't often get a chance to discuss those views directly. A Viewpoint article aims to bring those distinct views to the fore. For our first Viewpoint article, we have chosen the microbiome, and five experts consider the future of consortia-driven research, the limitations of the studies conducted to date and the direction for future microbiome research. If there any particular areas that you think it would be interesting to read about in a Viewpoint article, please do let us know, on Twitter or by e-mail. Your feedback, as ever, is very welcome. 